\title{
Randomized phase III trial of radiotherapy or chemoradiotherapy with topotecan and cisplatin in intermediate-risk cervical cancer patients after radical hysterectomy
}

Wenze Sun, Tao Wang, Fan Shi, Jiquan Wang, Juan Wang, Beina Hui, Yingbing Zhang, Jinli Lu, Hongwei Chen and Zi Liu*

\begin{abstract}
Background: In cervical cancer patients with intermediate-risk factors, the optimal adjuvant therapy is still controversial. We undertook a randomized trial (ClinicalTrials.gov Identifier: NCT01418859) to compare the efficacy and toxicity of concurrent chemoradiotherapy with topotecan and cisplatin with radiotherapy alone in intermediate-risk cervical cancer patients.

Methods: Eligible patients were randomly assigned to one of three treatment arms including arm A (radiotherapy only,RT), arm B(concurrent chemoradiotherapy only, CCRT), and arm C (concurrent chemoradiotherapy with following consolidation chemotherapy, CCRT $+C T$ ). All eligible patients completed external RT (IMRT or 3D-CRT), receiving 45-50Gy /25f uniformly to the pelvis. Concurrent chemotherapy regimen was topotecan $0.75 \mathrm{mg} / \mathrm{m}^{2}$ for days 1,2 and 3, followed by cisplatin $25 \mathrm{mg} / \mathrm{m}^{2}$ for days 1,2 and 3 . Three cycles of consolidation chemotherapy regimen was topotecan $1.5 \mathrm{mg} / \mathrm{m}^{2}$ for days 1 and 2, and $0.75 \mathrm{mg} / \mathrm{m}^{2}$ for day 3; followed by cisplatin $25 \mathrm{mg} / \mathrm{m}^{2}$ for days 1,2 and 3, repeated every 21 days. Adverse events of each group were investigated and compared.

Results: Thirty-nine patients enrolled onto the remaining regimens: 14 to RT, 15 to CCRT and 10 to CCRT + CT. Six patients (15.4\%) did not complete the protocol treatment. Hematologic toxicity was more frequent and more severe in the CCRT and CCRT + CT arms compared with the RT arm. The incidence of grade 3-4 neutropenia was significantly different statistically between the RT, CCRT and CCRT + RT groups (15.4\%, $46.7 \%$ and $100 \%$, respectively; $P=0.002$ ). Specially, three patients in CCRT $+C T$ arm of all six patients who did not complete the protocol treatment discontinued planned therapy because of persistent grade 4 neutropenia. However, there were no significant differences in grade 3-4 non-hematologic toxicities between the three groups(all $P>0.05$ ). Recurrence-free survival and overall survival of each group were not analyzed on account of a median follow-up of only 16 months.
\end{abstract}

Conclusions: Concurrent chemoradiotherapy with topotecan and cisplatin showed severe hematologic toxicity in intermediate-risk cervical cancer patients after radical hysterectomy. Thus, the study was closed ahead of schedule.

Trial registration: ClinicalTrials.gov Identifier: NCT01418859.

Keywords: Chemoradiotherapy, Topotecan, Cisplatin, Intermediate-risk, Cervical cancer

\footnotetext{
*Correspondence: liuzmail@163.com

Department of Radiation Oncology, First Affiliated Hospital of Xi'an Jiaotong University, 277 Yanta West Road, Yanta District, Xi'an 710061, China
} 


\section{Background}

Radical hysterectomy with pelvic lymphadenectomy has been widely accepted as treatment in patients with stage IB-IIA cervical cancer. However, the survival of patients with early stage cervical cancer after radical hysterectomy and pelvic lymphadenectomy depends on the presence or absence of several poor prognostic clinicopathologic risk factors. Positive or close resection margin, lymph node involvement, and parametrial invasion were recognized as high-risk factors while large tumor size, deep stromal invasion, and lymphovascular space invasion (LVSI) were regarded as intermediate-risk factors [1].

Radiation has been used as a postoperative adjuvant therapy to reduce recurrences in cervical cancer patients with both these intermediate and high risk factors [2-5]. Since Peters et al. showed a significant survival advantage with the use of concurrent chemoradiation rather than radiation in patients with high-risk cervical cancer [6]. Concurrent chemoradiation rapidly replaced radiation in the treatment of high-risk cervical cancer [6-8].

Although radiotherapy has been used as postoperative adjuvant therapy to reduce recurrence and improve survival in patients with intermediate risk tumors and the effectiveness of radiotherapy has been widely accepted, based on the results of a randomized study reported by Sedlis et al. [9]. Recent retrospective studies insisted that adjuvant radiotherapy may have local effects only, which are not effective in distant recurrence and have suggested the promising improvements of adding chemotherapy to radiation in patients with intermediate-risk factors [10-12]. Thus, two practical debates still remain regarding for the adjuvant treatment of the intermediate-risk group. First, is whether concurrent chemoradiotherapy (CCRT), which has been established as a standard adjuvant treatment of high-risk cervical cancer, is more beneficial than radiotherapy only for an intermediate-risk group. Second, cisplatin-based chemotherapy usually has been accepted as effective adjuvant therapy against cervical cancer. However, the optimal platinum-based combination treatments in adjuvant concurrent chemoradiation (CCRT) is still under investigation. Topotecan is an agent with antineoplastic activity on recurrent or metastatic cervical cancer and has been shown acceptable toxicity and good response rates in previous studies as a combined regimen with cisplatin $[13,14]$. Consequently, topotecan/ cisplatin was chosen as concurrent chemotherapy regimen in our study.

The aim of the present study (ClinicalTrials.gov Identifier: NCT01418859) was to investigate the efficacy and safety of CCRT with topotecan/cisplatin as adjuvant therapy after radical hysterectomy for cervical cancer patients with intermediate-risk factors.

\section{Methods}

\section{Eligibility}

Patients from First Affiliated Hospital of Xi'an Jiaotong University who had undergone radical surgery for clinical stage IB- IIA carcinoma of the cervix were enrolled in the study from September 2011 to August 2013. Radical hysterectomy and pelvic lymphadenectomy, with or without para-aortic lymphadenectomy was performed either via laparotomy or laparoscopy, according to the Gynecologic Procedures Manual of the GOG, which outlined minimum surgical requirements for study entry [15]. Patients who had biopsy-confirmed or were strongly suspected of para-aortic node metastasis on preoperative CT or MRI scan were not eligible. A total of 39 patients who had squamous carcinoma, adenocarcinoma, or adenosquamous carcinoma were eligible and all had histologically confirmed the following intermediate risk factors: greater than one-third stromal invasion, lymphovascular space involvement, or tumor size $\geq 4 \mathrm{~cm}$. Patients who had high-risk factors, such as positive pelvic lymph nodes, parametrial involvement, or positive surgical margins, were excluded. Registration was required within 4-6 weeks of surgery, with RT to begin within 3-5 working days of registration.

Patients were required to have an Eastern Cooperative Oncology Group performance status of 0 to 2 . The research was approved by Institutional Ethics Committee of First Affiliated Hospital of Xi'an Jiaotong University. The written informed consent was obtained from each patient.

\section{Treatment}

Following registration, patients were randomly assigned to one of three treatment arms including arm A (radiotherapy only), arm B (concurrent chemoradiotherapy only), and arm $\mathrm{C}$ (concurrent chemoradiotherapy with following consolidation chemotherapy).

All the patients underwent external beam radiation therapy to whole pelvis, but no brachytherapy. The external RT was given using IMRT or 3D-CRT with $\mathrm{x}$-ray energy of $10 \mathrm{MV}$ and consisted of 180-200 cGy per day on days 1 to 5 of each week, for a total of 25 fractions. Radiation dose ranged from 4500 to 5000 cGy. Radiation therapy was withheld if a patient had an absolute neutrophil count $<500 / \mathrm{mm}^{3}$, and delays of up to 5 days were also allowed in the event of radiation-related gastrointestinal or genitourinary toxicity.

One cycle of chemotherapy was administered at the second week concurrent with RT as follows:topotecan $0.75 \mathrm{mg} / \mathrm{m}^{2}$ intravenously during 30 minutes, followed by cisplatin $25 \mathrm{mg} / \mathrm{m}^{2}$ intravenously for days 1,2 and 3 .

Three cycles of consolidation chemotherapy were performed followed by first concurrent chemotherapy at the 6 th, 10th and 14th weeks. The regimen was topotecan 
$1.5 \mathrm{mg} / \mathrm{m}^{2}$ for days 1 and 2 , and $0.75 \mathrm{mg} / \mathrm{m}^{2}$ for day 3 ; followed by cisplatin $25 \mathrm{mg} / \mathrm{m}^{2}$ for day 1,2 and 3, repeated every 21 days.

The protocol is registered with ClinicalTrials.gov, number NCT01418859.

\section{Treatment modifications}

Dose adjustment was based on the greatest toxicity grade, using the National Cancer Institute Common Toxicity Criteria for Adverse Events (version 3). Chemotherapy was performed, providing the patient's absolute neutrophil count recovered to $\geq 1500 / \mathrm{mm}^{3}$ and platelets were $\geq 100,000 / \mathrm{mm}^{3}$ on the day of re-treatment. The cisplatin dose was decreased by $50 \%$ for grade 2 renal toxicity and held for the present cycle for grade 3 to 4 renal toxicity on the scheduled day of re-treatment. While topotecan was reduced by $20 \%$ for grade 3 and by $40 \%$ for grade 4 interval hematologic toxicity for the entire course of therapy. No dose reductions were allowed for grade 1 or 2 interval hematologic toxicity. Chemotherapy was also withheld until resolution of any grade 3 or 4 nonhematologic toxicity.

\section{Analysis}

Between the radiotherapy, chemoradiotherapy, chemoradiotherapy plus consolidation chemotherapy groups, the differences of clinicopathologic variables were evaluated using the chi-square test, Fisher's exact test, or linear by linear association method for categorical variables. Adverse event rates of the three groups were compared using Fisher's exact test. All analyses were performed using SPSS 17.0 software (SPSS Inc., Chicago, IL) and $P$ values less than 0.05 were considered significant.

\section{Results}

\section{Treatment and compliance}

Of the remaining 39 eligible patients (stage IB-IIA), 14 were randomly allocated to receive radiotherapy only (arm A), 15 were randomly allocated to receive chemoradiotherapy (arm B) and 10 were randomly allocated to receive chemoradiotherapy plus consolidation chemotherapy (arm C). Among patients randomly allocated to receive radiotherapy, chemoradiotherapy or chemoradiotherapy plus consolidation chemotherapy, patient characteristics were well-balanced (Table 1).

Fifteen percent of patients (6 of 39) did not complete protocol therapy. Three patients discontinued planned therapy in arm $C$ because of persistent grade 4 neutropenia toxicity. Other three patients also did not complete the therapy, including one in arm A because of costs of treatment and two in arm $C$ because of refusal to continue. Therefore, 33 patients, 13 in arm A, 15 in arm $\mathrm{B}$, and 5 in arm $\mathrm{C}$ completed treatment protocol and were analysed.
Table 1 Patient characteristics

\begin{tabular}{|c|c|c|c|c|}
\hline $\begin{array}{l}\text { Clinicopathologic } \\
\text { variables }\end{array}$ & $\begin{array}{l}\text { RT } \\
(N=13)\end{array}$ & $\begin{array}{l}\text { CCRT } \\
(N=15)\end{array}$ & $\begin{array}{l}\text { CCRT + CT } \\
(\mathrm{N}=5)\end{array}$ & $P$ value \\
\hline \multicolumn{5}{|l|}{ Age } \\
\hline$\leq 50$ & 3 & 4 & 1 & 0.558 \\
\hline$>50$ & 10 & 11 & 4 & \\
\hline \multicolumn{5}{|l|}{ Cell type } \\
\hline Squamous & 12 & 13 & 5 & 0.329 \\
\hline Non-squamous & 1 & 2 & 0 & \\
\hline \multicolumn{5}{|l|}{ Tumor grade } \\
\hline I & 1 & 1 & 0 & 0.583 \\
\hline$\|$ & 9 & 10 & 3 & \\
\hline III & 3 & 4 & 2 & \\
\hline \multicolumn{5}{|l|}{ Stage } \\
\hline IB1 & 1 & 2 & 0 & 0.782 \\
\hline IB2 & 8 & 9 & 3 & \\
\hline$\| \mathrm{A} 1$ & 2 & 3 & 1 & \\
\hline$\| \mathrm{A} 2$ & 2 & 1 & 1 & \\
\hline \multicolumn{5}{|l|}{ Risk factors } \\
\hline LTS only & 1 & 2 & 0 & 0.489 \\
\hline DSI only & 7 & 7 & 3 & \\
\hline LVSI only & 0 & 0 & 0 & \\
\hline LTS + DSI & 5 & 5 & 2 & \\
\hline LTS + LVSI & 0 & 0 & 0 & \\
\hline DSI + LVSI & 0 & 1 & 0 & \\
\hline LTS + DSI + LVSI & 0 & 0 & 0 & \\
\hline
\end{tabular}

Abbreviations: $R T$ radiotherapy, CCRT chemoradiotherapy, $C C R T+C T$ chemoradiotherapy plus consolidation chemotherapy, LTS large tumor size, DSI deep stromal invasion, LVSI lymphovascular space involvement.

Radiotherapy was withheld in 12 patients owing to grade 4 hematologic toxicity or for 5 to 9 days until neutropenia recovered to grade 2 . Chemotherapy was suspended because of grade 4 hematologic toxicity in 7 patients during consolidation chemotherapy. Specially, three patients discontinued planned therapy in arm $\mathrm{C}$ because of persistent grade 4 neutropenia.

Recurrence-free survival and overall survival of each group were not analyzed on account of a median followup of only 16 months.

\section{Toxicity}

Adverse events are summarized in Table 2. Hematologic toxicity was more frequent and more severe in the CCRT and CCRT + CT arms compared with the RT arm. Grade 3 and 4 neutropenia occurred in 46.7\% (7/15) of patients who received CCRT and $100.0 \%(5 / 5)$ of patients who received CCRT + CT but in only $15.4 \%(2 / 13)$ of those who received RT only $(P=0.002)$. Febrile 
Table 2 Adverse events by treatment

\begin{tabular}{|c|c|c|c|c|c|c|c|}
\hline \multirow[t]{2}{*}{ Adverse event } & \multicolumn{2}{|c|}{$\begin{array}{l}\mathrm{RT} \\
\text { (Grade), } \\
\mathrm{N}=13\end{array}$} & \multicolumn{2}{|c|}{$\begin{array}{l}\text { CCRT } \\
\text { (Grade), } \\
\mathrm{N}=15\end{array}$} & \multicolumn{2}{|c|}{$\begin{array}{l}\text { CCRT + CT } \\
\text { (Grade), } \\
\mathrm{N}=5\end{array}$} & \multirow[t]{2}{*}{$P$ value } \\
\hline & 3 & 4 & 3 & 4 & 3 & 4 & \\
\hline Neutropenia & 2 & 0 & 2 & 5 & 3 & 2 & 0.002 \\
\hline Anemia & 1 & 0 & 2 & 0 & 1 & 0 & 0.128 \\
\hline Thrombocytopenia & 1 & 0 & 3 & 1 & 1 & 1 & 0.079 \\
\hline Nausea/vomiting & 1 & 0 & 2 & 0 & 1 & 0 & 0.446 \\
\hline Diarrhea & 1 & 0 & 2 & 1 & 1 & 0 & 0.383 \\
\hline Genitourinary & 1 & 0 & 1 & 0 & 1 & 0 & 0.673 \\
\hline Neurotoxicity & 0 & 0 & 0 & 0 & 0 & 0 & 1 \\
\hline Renal & 0 & 0 & 0 & 0 & 0 & 0 & 1 \\
\hline Hepatic & 0 & 0 & 1 & 0 & 1 & 0 & 0.339 \\
\hline
\end{tabular}

Abbreviations: $R T$ radiotherapy, CCRT chemoradiotherapy,

$C C R T+C T$ chemoradiotherapy plus consolidation chemotherapy.

neutropenia occurred in $40.0 \%(2 / 5)$ of patients treated with CCRT + CT and $14.3 \%(1 / 7)$ of those who received CCRT. Grade 3 and 4 thrombocytopenia occurred in $26.7 \%(4 / 15)$ of patients who received CCRT and $40.0 \%$ $(2 / 5)$ of patients who received CCRT + CT versus only $7.7 \%(1 / 13)$ of those who received RT. $(P=0.079)$. Dose reductions were required for all the 5 patients in $\mathrm{CCRT}+\mathrm{CT}$ group in a subsequent cycle. However, there were no significant differences in grade 3 or 4 non-hematologic toxicities between the three groups. Diarrhea grades 3 and 4 were observed in 1 (7.6\%) patients of RT group, in $3(20.0 \%)$ patients of CCRT group and in 1 (20.0\%) patient of CCRT + CT group, respectively $(P=0.383)$. Genitourinary grades 3 and 4 occurred in only 1 patient in each arms $(P=0.673)$.

\section{Discussion}

Radical hysterectomy with pelvic lymphadenectomy has been widely accepted as the treatment in patients with IB-IIA cervical cancer. Because $10-20 \%$ of the patients recurred after radical surgery, efforts to reduce recurrences and improve survival in patients with cervical cancer have been made [11].

Since the beneficial effect of adjuvant concurrent chemoradiation for cervical patients with high-risk factors was proven in prospective studies from the Gynecologic Oncology Group (GOG) and the Radiation Therapy Oncology Group [3,6], concurrent chemoradiation is considered the optimum postoperative adjuvant therapy in patients with high-risk factors. However, in a Gynecologic Oncology Group study(GOG 92) that attempted to investigate the role of adjuvant radiotherapy (RT) in the intermediate-risk group, 277 cervical cancer patients with intermediate risk factors were randomized to the no further treatment (NFT) or RT group [9]. In those who met the GOG criteria, which defined the intermediate-risk group using a three-factor model of a combination of intermediate risk factors (capillary lymphatic space involvement, stromal invasion depth, and tumor size), a statistically significant reduction of risk of recurrence and an increase in recurrence free survival was found in the RT group compared with the NFT group. Since the efficacy of adjuvant radiation for patients with intermediate-risk factors were demonstrated in the previous trials $[5,9,11,12]$, radiation is the standard adjuvant therapy in patients with intermediate-risk factors. However, as shown in the GOG 92 trial, the recurrence rate of the intermediate-risk group was $27.9 \%$ in the NFT group, comparable to that of patients with highrisk factors. Furthermore, even though the recurrence rate was reduced with the use of adjuvant $\mathrm{RT}$, the rate of recurrence was still significant (15\%, 21 of 137 cases) in the RT group [11]. In addition, in the study of Tuipae $S$ et al., distant recurrence was still the major pattern of treatment failure after adjuvant radiotherapy in cervical cancer with intermediate risk factors [12].

Maybe the adjuvant chemoradiation have more beneficial effect over radiation only for patients with intermediate risk factors. An early study reported that, in patients with LVSI and deep stromal invasion, the 3-year RFS (recurrence-free survival) of the concurrent chemoradiation group was significantly greater than that of the radiation group [16]. Some retrospective studies in recent years also have showed the benefit of adding chemotherapy to radiation in patients with intermediaterisk factors and the levels of all toxicities were acceptable [17-20]. A statistically significant difference was found in the 3-year RFS rate among the no further treatment, RT, and CCRT groups $(67.5 \%, 90.5 \%$, and $97.5 \%$, respectively; $p<0.05$ ) in the study of Ryu SY et al. [18]. Okazawa $\mathrm{M}$ et al. reviewed the medical records of 316 patients with stage IB1-IIB cervical cancer who had been treated with adjuvant radiotherapy (RT) $(\mathrm{n}=124$, RT group) or adjuvant CCRT ( $\mathrm{n}=192$, CCRT group) after radical hysterectomy and found that CCRT was superior to RT with regard to recurrence rate and PFS in patents with 2 or more intermediate risk factors, while the patients with only 1 intermediate risk factor showed no survival benefit of CCRT over RT [20]. However, due to the lack of randomized trials, the benefit of concurrent chemoradiation over radiation in cervical cancer patients with intermediate-risk factors remains unclear.

Furthermore, adjuvant CCRT modalities for intermediate risk tumors have been debating. Weekly cisplatin or cisplatin plus 5-fluorouracil (5- FU) every 3 weeks has been widely used as the preferred treatment with CCRT in previous studies. Nevertheless, these standard regimens had some disadvantages [21,22]. For instance, the weekly cisplatin frequently caused a treatment delay due 
to hematologic toxicities and the requirement of a weekly visit to the hospital was inconvenient. In addition, 5-fluorouracil/cisplatin, a five-day regimen, caused more discomfort to patients and sometimes caused intractable nausea and vomiting. While previous studies using topotecan/cisplatin for patients with recurrent or metastatic cervical cancer have shown acceptable toxicity and good response rates. Thus, topotecan with cisplatin was chosen as concurrent chemotherapy regimen in our study.

Recently, cisplatin-based consolidation chemotherapy after CCRT has been reported to enhance local control and promote eradication of possible distant micrometastases in locally advanced cervical cancer by phase II studies [23-25]. These promsing results suggested that consolidation chemotherapy after primary CCRT may play a role independent of the radiotherapy in locally advanced cervical carcinoma. Furthermore, for high-risk patients after radical surgery, the continuation of chemotherapy (cisplatin $70 \mathrm{mg} / \mathrm{m}^{2}$ on day 1 and 5 -FU $1000 \mathrm{mg} / \mathrm{m}^{2} / \mathrm{d}$ for 5 days) for three to four cycles resulted in improved patient survival compared with the survival of patients who receive only one or two courses of chemotherapy $(P<0.03)$ in the study of Peters 3rd WA et al. [3]. It was not determined from their study whether the effect of the chemotherapy was as a radiation sensitiser or as a systemic chemotherapy with elimination of possible micrometastasis, or both. In contrast, Kim HS et al. found that consolidation chemotherapy after CCRT using paclitaxel and carboplatin failed to improve PFS and OS as compared to CCRT-only in patients with high-risk early-stage cervical cancer who underwent surgical treatment (3-year PFS, 62.7\% vs. $88.2 \%$, respectively; 3 -year OS, $90.9 \%$ vs. $97.3 \%$, respectively) [26]. However, is there any role for consolidation chemotherapy following CCRT in intermediate-risk cervical cancer patients? We designed arm $\mathrm{C}(\mathrm{CCRT}+\mathrm{CT})$ to examine whether the addition of consolidation chemotherapy to CCRT could improve the progressionfree survival and overall survival in patients with intermediate-risk factors after radical surgery.

On account of a median follow-up of only 16 months, RFS (recurrence-free survival) and OS (overall survival) of each group were not analyzed in the present study. Another issue that must be discussed is complications of these adjuvant therapies following radical surgery. Although cisplatin-based chemotherapy concurrent with RT is a standard treatment modality for advanced-stage cervical cancer, many physicians are still reluctant to treat the intermediate-risk group with CCRT. Because combined modalities are more likely to result in serious complications. As is known, radiotherapy after radical hysterectomy has been reported to be associated with high incidence of complications and many of these conditions are very tragic and irreversible and result in poor quality of life $[27,28]$. Barter et al. observed that $30 \%$ of patients treated with radiation after radical hysterectomy had serious complications, such as gastrointestinal and genitourinary complications and leg lymphedema et al. [28]. Meanwhile, the major toxicities of combined chemotherapy were bone marrow depression, mild to moderate gastrointestinal troubles, mild to moderate nephrotoxicities, total alopecia (in etoposidecisplatin group), relatively low incidence of neurotoxicities and hepatotoxicities, rare incidence of pulmonary and cardiac toxicities and some reported secondary malignancies (acute leukemia and some solid tumors). In previous studies the levels of all these toxicities of combined chemotherapy were acceptable and most are reversible and transient [17-20]. Nevertheless, the results of the present study found that hematologic toxicity was significantly more frequent and more severe in the CCRT and CCRT + CT arms compared with the RT arm. Grade 3 and 4 neutropenia occurred in $46.7 \%(7 / 15)$ of patients who received CCRT and $100.0 \%(5 / 5)$ of patients who received CCRT + CT but in only $15.4 \%(2 / 13)$ of those who received RT only $(P=0.002)$. Especially, three patients discontinued planned therapy in arm $C$ because of persistent grade 4 neutropenia toxicity. Although topotecan with cisplatin has been shown acceptable toxicity and good response rates in recurrent or metastatic cervical cancer, similar to the study of Long HJ 3rd et al., which reported that the combination of topotecan-cisplatin resulted in substantial neutropenia ( $70 \%$ vs $1.4 \%)$ compared with single cisplatin agent, and nearly $18 \%$ of patients in the topotecan-cisplatin arm experienced febrile neutropenia in patients with advanced (stage IVB), recurrent or persistent carcinoma of the uterine cervix, topotecan plus cisplatin chemotherapy concurrent with RT in our study also showed severe hematologic toxicity [14]. Therefore, we closed the clinical trial ahead of schedule.

Although our study showed severe hematologic toxicity of concurrent chemoradiation with topotecan and cisplatin in safe dose to radiation in patients with intermediate-risk factors, the efficacy and toxicity are still unclear. As we mentioned in the results, the number of patients in the different group is small (14 in group A, 15 in group B and 10 in group C) because the study closed ahead of schedule and the media follow-up time is only 16 months. In spite of these limitations, due to topotecan has been identificated as an antineoplastic agent with cisplatin in cervival cancer, a trial with optimal dose, sufficient size and long term follow-up would be necessary to demonstrate the advantage of concurrent chemoradiation in patients with intermediaterisk factors. 


\section{Conclusions}

To conclude that chemoradiotherapy maybe the better adjuvant therapy than radiation in patients with intermediate-risk factors, but the benefit of chemoradiotherapy should overweigh the cost of adverse effects. The present study showed severe hematologic toxicity in the chemoradiotherapy with topotecan and cisplatin groups and was closed ahead of schedule. Therefore, the optimal platinum-based adjuvant chemoradiotherapy in cervical cancer patients with intermediate risk factors is still under investigation especially and the final conclusion regarding the role of chemoradiotherapy should be withheld.

\section{Abbreviations}

RT: Radiotherapy; CCRT: Concurrent chemoradiotherapy; CCRT + CT: Concurrent chemoradiotherapy with following Consolidation chemotherapy; IMRT: Intensity modulated radiation therapy; 3D-CRT: 3-dimensional conformal radiotherapy; LTS: Large tumor size; DSI: Deep stromal invasion; LVSI: Lymphovascular space invasion; GOG: Gynecologic Oncology Group; CT: Computed tomography; MRI: Magnetic resonance imaging; NFT: No further treatment; RFS: Recurrencefree survival; OS: Overall survival.

\section{Competing interests}

The authors declare that they have no competing interests.

\section{Authors' contributions}

WS guarantees the integrity of the entire study and drafted the manuscript. TW, FS, JW and BH collected medical records. JW and TW performed statistical analyses. FS and $J \mathrm{~L}$ searched and arranged the references. ZL and $\mathrm{HC}$ modified the manuscript. ZL conceived the study, participated in its design and coordination, and completed the final proofreading of the manuscript. All authors have read and approve the final manuscript.

\section{Authors' information}

Address of the submitting author: Wenze Sun at 277 Yanta West Road, Yanta District, Xi'an, 710061, China.

\section{Acknowledgements}

This study was financially supported by the Department of Health Key Program of Shaanxi Province (No.2010A02)

Received: 24 October 2014 Accepted: 23 April 2015

Published online: 04 May 2015

\section{References}

1. Bidus MA, Elcas JC. Cervical and vaginal cancer. In: Berek JS, editor. Novak EBerek \& Novak's gynecology. 14th ed. Philadelphia: Lippincott Williams \& Wilkins; 2007. p. 1403-56.

2. Hacker NF, Friedlander ML, Berek JS. Cervical cancer. In: Hacker NFBerek \& Hacker's gynecologic oncology. 5th ed. Philadelphia: Lippincott Williams \& Wilkins; 2010. p. 341-95.

3. Peters 3rd WA, Liu PY, Barrett 2nd RJ, Stock RJ, Monk BJ, Berek JS, et al. Concurrent chemotherapy and pelvic radiation therapy compared with pelvic radiation therapy alone as adjuvant therapy after radical surgery in high-risk early-stage cancer of the cervix. J Clin Oncol. 2000;18:1606-13.

4. Delgado G, Bundy BN, Fowler Jr WC, Stehman FB, Sevin B, Creasman WT, et al. A prospective surgical pathological study of stage I squamous carcinoma of the cervix: a Gynecologic Oncology Group Study. Gynecol Oncol. 1989;35:314-20.

5. Delgado G, Bundy B, Zaino R, Sevin BU, Creasman WT, Major F. Prospective surgical-pathological study of disease-free interval in patients with stage IB squamous cell carcinoma of the cervix: a Gynecologic Oncology Group study. Gynecol Oncol. 1990:38:352-7.

6. Rose PG, Bundy BN, Watkins EB, Thigpen JT, Deppe G, Maiman MA, et al. Concurrent cisplatin-based radiotherapy and chemotherapy for locally advanced cervical cancer. N Engl J Med. 1999;340:1144-53.
7. Morris M, Eifel PJ, Lu J, Grigsby PW, Levenback C, Stevens RE, et al. Pelvic radiation with concurrent chemotherapy compared with pelvic and para-aortic radiation for high-risk cervical cancer. $N$ Engl J Med. 1999;340:1137-43.

8. Ryu HS. Concurrent chemoradiotherapy in cervical cancer (a new paradigm in cervical cancer treatment). Yonsei Med J. 2002;43:749-53.

9. Sedlis A, Bundy BN, Rotman MZ, Lentz SS, Muderspach LI, Zaino RJ. A randomized trial of pelvic radiation therapy versus no further therapy in selected patients with stage IB carcinoma of the cervix after radical hysterectomy and pelvic lymphadenectomy: a gynecologic oncology group study. Gynecol Oncol. 1999;73:177-83.

10. Ho CM, Chien TY, Huang SH, Wu CJ, Shih BY, Chang SC. Multivariate analysis of the prognostic factors and outcomes in early cervical cancer patients undergoing radical hysterectomy. Gynecol Oncol. 2004;93:458-64.

11. Rotman M, Sedlis A, Piedmonte MR, Bundy B, Lentz SS, Muderspach LI, et al. A phase III randomized trial of postoperative pelvic irradiation in Stage IB cervical carcinoma with poor prognostic features: follow-up of a gynecologic oncology group study. Int J Radiat Oncol Biol Phys. 2006:65:169-76.

12. Tuipae $S$, Yanaranop $M$, Oniem $N$. Role of adjuvant radiotherapy after radical hysterectomy in node-negative stage IB-IIA cervical cancer with intermediate risk factors. J Med Assoc Thai. 2012;95 Suppl 3:S117-24.

13. Muderspach LI, Blessing JA, Levenback C, Moore Jr JL. A Phase II study of topotecan in patients with squamous cell carcinoma of the cervix: a gynecologic oncology group study. Gynecol Oncol. 2001;81:213-5.

14. Long 3rd HJ, Bundy BN, Grendys Jr EC, Benda JA, McMeekin DS, Sorosky J, et al. Randomized phase III trial of cisplatin with or without topotecan in carcinoma of the uterine cervix: a Gynecologic Oncology Group Study. J Clin Oncol. 2005;23:4626-33.

15. Whitney CW, Spirtos N. Gynecologic Oncology Group Surgical Procedures Manual. January 2005. Available at: https://gogmember.gog.org/manuals/ pdf/surgman.pdf. Accessed July 1, 2005.

16. Soisson AP, Soper JT, Clarke-Pearson DL, Berchuck A, Montana G, Creasman WT. Adjuvant radiotherapy following radical hysterectomy for patients with stage IB and IIA cervical cancer. Gynecol Oncol. 1990;37:390-5.

17. Kim K, Kang SB, Chung HH, Kim JW, Park NH, Song YS. Comparison of chemoradiation with radiation as postoperative adjuvant therapy in cervical cancer patients with intermediate-risk factors. Eur J Surg Oncol. 2009;35:192-6.

18. Ryu SY, Park SI, Nam BH, Cho CK, Kim K, Kim BJ, et al. Is adjuvant chemoradiotherapy overtreatment in cervical cancer patients with intermediate risk factors? Int J Radiat Oncol Biol Phys. 2011;79:794-9.

19. Lee TY, Jeung YJ, Lee CJ, Kim HY, Kim SH, Kim WG. Promising treatment results of adjuvant chemotherapy following radical hysterectomy for intermediate risk stage 1B cervical cancer. Obstet Gynecol Sci. 2013;56:15-21.

20. Okazawa M, Mabuchi S, Isohashi F, Suzuki O, Yoshioka Y, Sasano T, et al. Impact of the addition of concurrent chemotherapy to pelvic radiotherapy in surgically treated stage IB1-IIB cervical cancer patients with intermediaterisk or high-risk factors: a 13-year experience. Int J Gynecol Cancer. 2013;23:567-75.

21. Cetina L, Rivera L, Hinojosa J, Poitevin A, Uribe J, López-Graniel C, et al. Routine management of locally advanced cervical cancer with concurrent radiation and cisplatin. Five year results. BMC Womens Health. 2006;6:3.

22. Lee TS, Kang SB, Kim YT, Park BJ, Kim YM, Lee JM, et al. Chemoradiation with paclitaxel and carboplatin in high-risk cervical cancer patients after radical hysterectomy: a Korean Gynecologic Oncology Group study. Int J Radiat Oncol Biol Phys. 2013;86:304-10.

23. Vrdoljak E, Prskalo T, Omrcen T, Situm K, Boraska T, Frleta Ilić N, et al. Concomitant chemobrachyradiotherapy with ifosfamide and cisplatin followed by consolidation chemotherapy in locally advanced squamous cell carcinoma of the uterine cervix: results of a phase II study. Int J Radiat Oncol Biol Phys. 2005;61:824-9.

24. Zhang MQ, Liu SP, Wang XE. Concurrent chemoradiotherapy with paclitaxel and nedaplatin followed by consolidation chemotherapy in locally advanced squamous cell carcinoma of the uterine cervix: preliminary results of a phase II study. Int J Radiat Oncol Biol Phys. 2010;78:821-7.

25. Choi CH, Lee YY, Kim MK, Kim TJ, Lee JW, Nam HR, et al. A matched-case comparison to explore the role of consolidation chemotherapy after concurrent chemoradiation in cervical cancer. Int J Radiat Oncol Biol Phys. 2011;81:1252-7. 
26. Kim HS, Kim MK, Kim HJ, Han SS, Kim JW. Phase II Study of Consolidation Chemotherapy after Adjuvant or Primary Concurrent Chemoradiation Using Paclitaxel and Carboplatin to Treat High-Risk Early-Stage or Locally Advanced Cervical Cancer. Cancer Res Treat. 2012:44:97-103.

27. Dische S. Radiotherapy of cervical cancer. Clin Obstet Gynaecol. 1985;12:203-27.

28. Barter JF, Soong SJ, Shingleton HM, Hatch KD, Orr Jr JW. Complications of combined radical hysterectomy-postoperative radiation therapy in women with early stage cervical cancer. Gynecol Oncol. 1989;32:292-6.

Submit your next manuscript to BioMed Central and take full advantage of:

- Convenient online submission

- Thorough peer review

- No space constraints or color figure charges

- Immediate publication on acceptance

- Inclusion in PubMed, CAS, Scopus and Google Scholar

- Research which is freely available for redistribution 